\title{
Endogenous Preferential Treatment in Centralized Admissions
}

\author{
Y. Stephen Chiu and Weiwei Weng*† \\ University of Hong Kong
}

September 12, 2007

\begin{abstract}
A large portion of school and college places in the world are allocated through centralized admissions schemes. In this paper, we study a model of centralized admissions with the feature that schools are allowed to pre-commit to admitting qualified applicants who rank them as top choice over more qualified applicants who do not. We identify two motives for doing so. A less popular school may use the pre-commitment to steal applicants who otherwise would not choose it as their top choice (stealing motive); a popular school may use the pre-commitment to prevent its own applicants from being stolen (preemptive motive). We show the conditions for such motives to exist, and in doing so, illustrate the roles of school popularity, difference in schools' preferences, and relative school size.

Keywords: centralized admissions, commitment, endogenous preferential treatment, manipulation
\end{abstract}

\section{Introduction}

A significant number of school and college places are allocated through centralized admission schemes. In places where such schemes exist, instead of filing separate applications to different schools, students need to file only a single application (a priority list) through which they indicate their preferences among schools that they are interested in; schools then

${ }^{*}$ Chiu (corresponding author): School of Economics and Finance, 918 K. K. Leung Building, University of Hong Kong, Pokfulam Road, HONG KONG. Phone: 2859-1056. Fax: 2548-1152. Email: schiu@econ.hku.hk. Weng: School of Economics and Finance, University of Hong Kong. Email: www19830@hotmail.com.

${ }^{\dagger}$ We wish to thank Atila Abdulkadiroğlu, Steve Ching, Eric Chou, Wing Suen, and seminar participants at Academia Sinica, National Tsing Hua University, Tsing Hua university, Central University of Economics and Finance, and University of Hong Kong for comments. Any remaining errors are, of course, our own responsibility. Financial support from Hong Kong Research Grant Council (HKU742407H) is gratefully acknowledged. 
allocate their places in accordance with a strict protocol, taking into account applicants' reported preferences as well as all other available information. Examples abound. It is common for commonwealth countries to assign their university places in this manner based on students' performance in public examinations (e.g., UK and its former colony, Hong Kong). China runs a gigantic national college admissions programme in which a few million college places are allocated every year (Table 21-2, Chinese Statistical Yearbook, 2006). In the US, school choice programs have become popular since the late 80's through which public junior school places are assigned. Unlike the traditional system in which school places are assigned strictly according to where students reside, the new school choice programs allow a much greater variety of schools for students' choosing (see, e.g., Abdulkadiroğlu and Sőnmez, 2003). ${ }^{1}$

In this context of centralized admissions, we are interested in a phenomenon in which a school gives preferential treatment to applicants who rank it as top choice over more qualified applicants who do not. Such preferential treatment may be an intrinsic feature of the admissions scheme being used. For instance, in the so called Boston admission scheme (Ergin and Sönmez, 2006; Abdulkadiroğlu, Pathak, Roth, and Sőnmez, 2005, 2006), school places are first assigned according to applicants' top choice. This means that an applicant failing to get into her top choice will also fail in getting into her second choice school if the latter school has already filled up its places with applicants ranking it as their top choiceeven though the applicant may be much better than many of the latter. In other cases, however, the preferential treatment may be just a deliberate choice of the school, rather than an intrinsic feature of the admissions scheme. In China, a university normally deduces 30 to 50 points of public examination score ${ }^{2}$ from an applicant who does not rank it as top choice when comparing her vis-a-vis other applicants who do; alternatively, the university may pre-set a quota of $5 \%$, say, for non-top choice applicants. This is so despite the fact that China' admissions scheme has no stipulations of such. It is this second type of preferential treatment that our paper will focus on.

Regarding why schools may deliberately choose to adopt the practise, we can think of two reasons. First, students' motivations and commitments are important to success in their studies, as is true in other endeavours. If a school cares about the success of its students in studies and future career, it may want to give preferential treatment during admissions to those who are genuinely more enthusiastic. In contrast with this motivational consideration, a school may adopt the policy out of a strategic consideration. If preferential treatment helps sway applicants to specify the school as their top choice and if this effect is strong enough, the school may eventually admit a better group of students. Thus, it is in the

\footnotetext{
${ }^{1}$ It is worthwhile pointing out that, in each place mentioned, alongside with the centralized admissions schemes, there are also separate channels (such as early admissions programmes) through which students can get admitted. These channels normally play an auxillary role, allocating a much smaller number of places each year. We will ignore these alternative channels in our analysis.

${ }^{2}$ Based on raw public exam score that ranges from a low of zero to a high of 750 .
} 
interest of the school to adopt the practise despite no intrinsic interest in each applicant's ranking of schools. In this paper we assume this latter strategic consideration, rather than the former motivational consideration. We think that this is operationally easier and, more importantly, closest to standard assumptions in this literature, allowing us a benchmark for comparison and with which to start further studies. ${ }^{3}$

The baseline model that we study is the Gale-Shapley mechanism (Gale and Shapley 1962), but we allow schools to pre-commit to admitting qualified applicants who rank it as their top choice. We call such a policy as the immediate acceptance (IA) policy, in contrast with the deferred acceptance (DA) policy inherent in the Gale-Shapley mechanism. ${ }^{4}$ More specifically, we consider a three-stage game. First, schools - or school place offering unitsannounce publicly if they pre-commit to an IA policy. Then, applicants report their rankings of schools, followed by assignment of places in accordance with the Gale-Shapley mechanism subject to constraints of schools' pre-commitments. Throughout the paper, we assume that, albeit strategic, schools are credulous; it is so perhaps because schools are honest, or simply because reputational concerns are strong enough as an enforcement device.

Our first finding is a neutrality result, which roughly says the following. So long as the outcome in the game in which all schools have DA policies is deterministic and commonly known, no schools would gain from adopting IA. Exactly the same outcome, in terms of who enters what school, would result. Simple as it seems, this result suggests that uncertainty is essential for understanding the role of IA behind its strategic use. In light of this, we study two types of uncertainty: demand uncertainty and ranking uncertainty.

In the demand uncertainty model, applicants are uncertain about others' genuine demands, i.e., others' preferences for places at different schools. This captures scenarios in which applicants may not be sure of the latest fads regarding whether computer science or business, say, is hot this year. In the ranking uncertainty model, applicants do not know how well they are ranked among themselves. This model captures the scenario in which applicants are required to apply before learning the exam results or even before taking examinations. To a certain extent, it also captures the scenario in which applicants are grouped into large indifferent classes because of coarse public examination scores; uncertainty about the tie-breaking outcome can be viewed as uncertainty in the rankings of applicants among themselves. ${ }^{5}$

By studying the two models, we identify two motives for pre-commitment. A school

\footnotetext{
${ }^{3}$ Evidence of the motivational consideration, from educators and counselors, is not difficult to find. After a change in the New York city's school choice program in 2004, an administrator complained that the information about how an applicant ranked a school was no longer available to the school and argued that it was important that the information be released. He said, "we want the students that want us most." (New York Times, November 29, 2004) We would like to thank Atila Adulkadiroglu for pointing out this source to us.

${ }^{4}$ We do not consider other types of preferential treatments because the one that we study is the most extreme one and presumably serves as the best starting point.

${ }^{5}$ The GCE exam, or its equivalence, that is normally taken in commonwealth countries gives only a letter grade or subgrade for each course taken. This leads to large indifferent classes.
} 
may use the IA to steal applicants who otherwise would not have made it their top choice (the stealing motive). A less popular school is more likely to have a stealing motive when it is not too inferior to a popular school in terms of the utility that enrolled students can obtain. On the other hand, rarely will a popular school have this motive. Under more restricted conditions, the popular school does have a motive to use IA (the preemptive motive). Although not helping to steal applicants, the IA is attractive because it can prevent the less popular school from using IA and whereby protecting the popular school from being hurt. We show the conditions for such motives to exist and relate them to school popularity, difference in schools' preferences, and relative school size. We also find that being able to influence applicants' behavior need not imply an improvement in the school's average student quality. IA can be influential yet self-defeating. Although our results are obtained in a two school setting, we think that the basic insights identified here should prevail in a more general setting.

The paper by Gale and Shapley has generated a large literature on matching and market design. Later work shows that under the deferred acceptance mechanism, some schools may have an incentive to misrepresent their preferences (Roth 1989; Roth and Sotomayor 1990). Sönmetz (1997) show that hospitals may benefit from misreporting their capacities; the same can be said about schools because of the similarity between school-student matching and hospital-medical intern matching. Despite this, in all of these studies, applicants still have truthful reporting as their dominant strategy. Abdulkadiroğlu and Sonnmez (2003) are the first to extend the studies to school choice problems in which schools are neither economic agents nor strategic. Reforms in real world social choice problems are being implemented in Boston and New York City. The paper on Boston school choice problem by Ergin and Sonnmetz (2006) is closest to us. There they characterize the equilibrium for a centralized admissions scheme in which preferential treatment is an intrinsic feature, rather than school's strategic choice. Issues such as the presence of large indifferent groups - a quite common feature that is ignored in earlier literature which is limited to strict preferenceshas prompted theoretical studies on the role of different tie-breaking rules and designs of better mechanisms (see Abdulkadiroğlu, Pathak and Roth 2006 and Erdil and Ergin, forthcoming). ${ }^{6}$

The rest of the paper is organized as follows. Section 2 describes the basic model in which the IA policy is irrelevant. Section 3 describes a modified model with demand uncertainty and analyzes the case of homogenous school preferences, and Section 4 generalizes the results to heterogeneous school preferences. Section 5 studies a model in which each applicant is uncertain about his or her relative ranking with respect to other applicants. Section 6 presents a discussion and Section 7 concludes the paper.

\footnotetext{
${ }^{6}$ In addition to school choice and college admission problems, mechanisms like those of Gale and Shapley have been put into practice in, for instance, the resident program in the US, where medical residents are assigned hospital places (Roth and Rothblum, 1984; Roth and Peranson 1999; Roth 1991).
} 


\section{Model}

A mass of applicants of size $N$ are competing to enter two schools. Each applicant is indexed by an ability attribute pair $\left(y_{1}, y_{2}\right)$, where $y_{1}$ may be her math score and $y_{2}$ her language score, for example. $\left(y_{1}, y_{2}\right)$ 's are distributed over $[0,1] \times[0,1]$ with a cumulative function $F$, which is continuous and has no mass point. Each applicant's ability pair, as well as, $F$, is commonly known, and as such she knows her exact ranking vis-a-vis other applicants. Given attribute pair $\left(y_{1}, y_{2}\right)$, a fraction $\mu_{1}$ of applicants view school 1 as the better school: the utility of attending the two schools is, respectively, $u_{1}$ and $u_{2}$, where $u_{1}>u_{2}>0$; a fraction, $\mu_{2}=1-\mu_{1}$, of applicants view school 2 as better: the utility of attending the two schools are $v_{1}$ and $v_{2}$, where $0<v_{1}<v_{2}$. We call the former group of applicants type 1 applicants and the second group type 2 applicants.

The two schools have places, $s_{1}$ and $s_{2}$, where $s_{1}+s_{2}<N$. We assume that school 1 is relatively more popular, i.e., $\mu_{1} / s_{1}>\mu_{2} / s_{2}$. Occasionally, we will call school 1 the popular school and school 2 the less popular school. School $i$ 's preferences are represented by a parameter $\alpha_{i} \geq 0, i=1,2$. School $i$ strictly prefers applicant $a$ with attribute pair $\left(y_{1}^{a}, y_{2}^{a}\right)$ over applicant $b$ with attribute pair $\left(y_{1}^{b}, y_{2}^{b}\right)$ if and only if $y_{1}^{a}+\alpha_{i} y_{2}^{a}<y_{1}^{b}+\alpha_{i} y_{2}^{b}$; hence, the smaller the attribute pair the better. We assume that, without loss of generality, $\alpha_{1} \leq \alpha_{2}$, i.e., school 1 places more emphasis on attribute 1 than on attribute 2 as compared with school 2. When $\alpha_{1}=\alpha_{2}$, the two schools have identical preferences; when $\alpha_{1}<\alpha_{2}$, the two schools may differ as to which of two applicants is better. Given a group of applicants characterized by cumulative function $H\left(y_{1}, y_{2}\right)$, their average quality for school $i$ is

$$
\frac{\int_{[0,1] \times[0,1]}\left(y_{1}+\alpha_{i} y_{2}\right) d H\left(y_{1}, y_{2}\right)}{\int_{[0,1] \times[0,1]} d H\left(y_{1}, y_{2}\right)} .
$$

Given two groups of applicants with the same mass, characterized by cumulative functions $H_{1}\left(y_{1}, y_{2}\right)$ and $H_{2}\left(y_{1}, y_{2}\right)$, school $i$ prefers the former group to the latter group if and only if, for school $i$, the former group's average quality is better (i.e., is lower) than that of the latter group.

A centralized admissions scheme in the fashion of Gale and Shapley (1962) is in place; each applicant is required to submit a priority list of schools - indicating whether she regards school 1 as her first choice and school 2 as her second choice or vice versa. Before the centralized admissions scheme actually operates, schools publicly announce which policy to use: a deferred acceptance (DA) policy or an immediate acceptance (IA) policy. Under the former, the school ranks applicants based solely on their quality attributes and processes the applications strictly in accordance with the Gale-Shapley mechanism. Under the latter, while still using the centralized admissions scheme, the school is committed to admitting applicants who specify it as their top choice up to its capacity. We assume that schools 
are honest so that the commitment is genuine and credible. The game is identical to the Gale-Shapley mechanism except that schools may pre-commit to an IA policy.

We use a pair $(k, l)$, where $k, l=D A, I A$, to represent the policies used by the two schools. Under (DA,DA), there exists an equilibrium in which all type 1 applicants report school 1 as their top choice and all type 2 applicants report school 2 as their top choice. The two schools then admit according to the standard Gale-Shapley procedure. We call the strategies the truthful strategies, the equilibrium the truthful equilibrium, and the outcome (in terms of who goes to what school) the truthful outcome. Making use of (1), we use $A Q_{i}(k, l)$ to denote school $i$ 's equilibrium average quality under policy pair $(k, l)$, where $i=1,2$, and $k, l=D A, I A$.

\subsection{An Irrelevance Result}

We first report an irrelevance result (all proofs are relegated in the Appendix).

Proposition 1 Suppose the truthful outcome under (DA,DA) is deterministic (no tie-breaking rule is used in determining the truthful outcome) and is commonly known. Then, under any policy pair $(i, j)$ where at least one of $i$ and $j$ equals $I A$, the unique equilibrium outcome is the truthful outcome.

Basically, foreseeing the impact of the IA policy, every applicant who enters some school in the truthful equilibrium now puts that school - the applicant knows what the school is - as his or her top choice. As such, the applicant succeeds in getting into the school, and the effect of the IA policy is completely neutralized. It is clear that this constitutes an equilibrium. ${ }^{7}$ Despite its success in affecting applicants' reporting, the IA policy does not bring improvement to the school in terms of the average quality of admitted applicants. This irrelevance result is in fact very general and does not depend on the assumptions of two schools and a continuum of applicants. The intuition is that, without uncertainty, applicants are able to replicate the same outcome by optimally misreporting their preferences. We will next turn to two models of uncertainty that make IA relevant.

\section{Demand Uncertainty with Identical School Prefer- ences $\left(\alpha_{1}=\alpha_{2}\right)$}

Here we assume that applicants are uncertain about the state of the world that determines applicants' preferences. We call this a model of demand uncertainty. There are two states

\footnotetext{
${ }^{7}$ The uniqueness of equilibrium comes from the stability of the truthful outcome, a well known result in the Gale-Shapley mechanism.
} 
of the world, state 1 and state 2 , with respective probabilities, $\pi_{1}$ and $\pi_{2} \equiv 1-\pi_{1}$. We assume that

$$
\mu_{1}^{1} / \mu_{2}^{1}>\mu_{1}^{2} / \mu_{2}^{2}>s_{1} / s_{2}
$$

where $\mu_{i}^{j} \in(0,1)$ is the fraction of applicants who prefers under state $j$ school $i$ to the other school. That is, school 1 is still more popular than school 2 under each state, but its popularity is less overwhelming under state 2. We study the same game described in the last section, except that, prior to the game, there is an additional stage in which the true state is realized (but not revealed to agents). We assume that schools are (von Neunman Morgenstern) expected average quality minimizers, i.e., school $i$ 's objective is to minimize $A Q_{i} \equiv \pi_{1} A Q_{i}^{1}+\pi_{2} A Q_{i}^{2}$, where $A Q_{i}^{j}$ is school $i$ 's average quality in state $j$.

\subsection{Unilateral Incentive}

To see why demand uncertainty may enable IA to become relevant, we focus on the simplest case in which $\alpha_{1}=\alpha_{2}=0$ : both schools have identical preferences and do not care about the $y_{2}$ attribute. We further assume that $y_{1}$ is uniformly distributed. Under (DA,DA), type $i$ applicants will specify school $i$ as their top choice, $i=1,2$. Because of (2), in equilibrium, school 1 will only admit type 1 applicants, setting a cutoff value, $c_{1}^{1}=s_{1} / \mu_{1}^{1} N$, in state 1 and a more lenient cutoff value, $c_{1}^{2}=s_{1} / \mu_{1}^{2} N$, in state 2. School 2, on the other hand, sets the same cutoff value, $c_{2}^{1}=c_{2}^{2}=\left(s_{1}+s_{2}\right) / N$, in both states, admitting all type 2 applicants satisfying this condition as well as all type 1 applicants satisfying this condition who have been declined by school 1 (see Figure 1).

Given this benchmark, it is transparent that school 2 may want to use IA, for the purpose of altering the behavior of those type 1 applicants with $y_{1} \in S \equiv\left(s_{1} / \mu_{1}^{1} N, s_{1} / \mu_{1}^{2} N\right)$ (i.e., applicants in Region A in Figure 1), who are uncertain about which school they will enter under (DA,DA). If $\pi_{2} u_{1}<u_{2}$, there no longer exists a truthful equilibrium under (DA,IA), for the following reason. Given that all others follow their truthful strategies, any type 1 applicant with $y_{1} \in S$ will benefit from a unilateral deviation to choosing school 2 as her top choice. As such, the applicant is guaranteed a place in school 2 in both states, resulting in a utility of $u_{2}$. By following the truthful strategy, however, the applicant will be admitted by school 1 in state 2 and not by any school in state 1 , resulting in a lower utility of $\pi_{2} u_{1}$.

It is easy to verify that a non-truthful equilibrium exists in which (i) all type 1 applicants with $y_{1} \in S$ specify school 2 as their top choice, and (ii) all type 1 applicants with $y_{1}<$ $s_{1} / \mu_{1}^{1} N$, as well as all type 2 applicants, report truthfully (other applicants' strategies are inconsequential). In this equilibrium, the state 1 outcome is exactly the same as in the truthful equilibrium. Under state 2, nonetheless, school 2 is able to admit the type 1 applicants with $y_{1} \in S$, setting a more stringent cutoff at $y_{1}=\left(s_{1}+s_{2}\right) / N-x$, where 


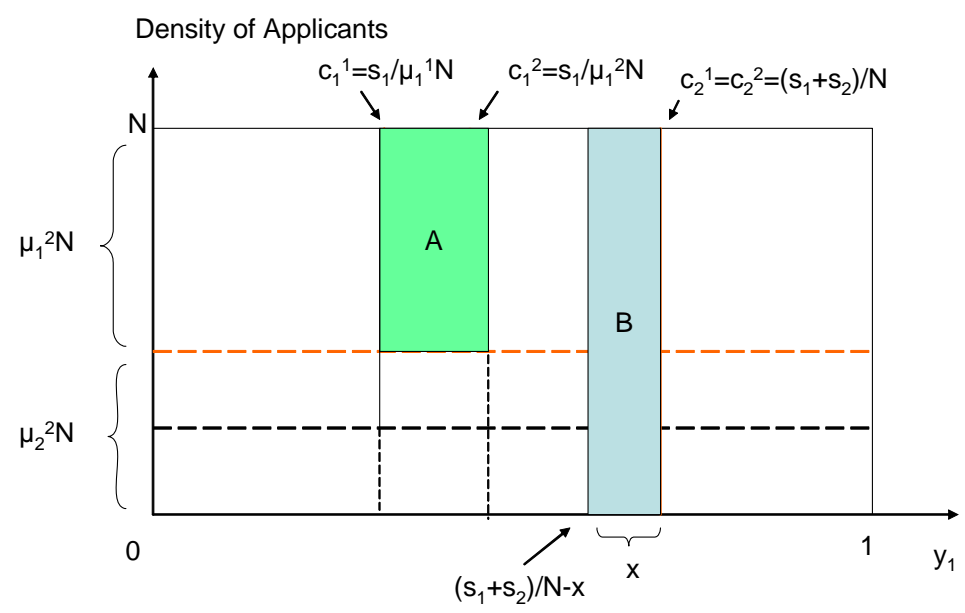

Figure 1: Under (DA,DA), the two schools set cutoffs, $c_{1}^{j}$ and $c_{2}^{j}$, in state $j, j=1,2$. In case $\pi_{2} u_{1}<u_{2}$, by switching to IA, school 2 steals in state 2 type 1 applicants in Region A from school 1, giving up applicants in Region B whereby.

$x \equiv\left(s_{1} / N\right)\left(1-\mu_{1}^{2} / \mu_{1}^{1}\right)$; diagrammatically, school 2 replaces students in Region B, ie., those applicants with $y_{1} \in T \equiv\left(\left(s_{1}+s_{2}\right) / N-x,\left(s_{1}+s_{2}\right) / N\right)$, by better students in Region A (see Figure 1). Thus, school 2 is strictly better off.

In other words, through the IA policy, school 2 is able to steal good type 1 students from school 1, and this is why school 2 may benefit from the policy. The stealing effect does not always exist, however. When $\pi_{2} u_{1}>u_{2}$, school 2's IA policy is unable to persuade type 1 applicants with $y_{1} \in S$ to make school 2 their top choice. As a result, neither are these students stolen by school 2 in state 2 , even worse, because of the pre-commitment, nor are they admitted by school 2 in state 1 - school 2 has to admit poorer applicants to fill the vacancies. School 2 is thereby worse off as a whole, and the IA policy is self-defeating. ${ }^{8}$

The analysis of the effect of school 1's IA is more straightforward. Since under (DA,DA) school 1 already sets a more stringent admissions requirement, those type 2 applicants whom school 1 is interested in stealing must be good enough to enter school 2 - their preferred school - and have no incentive to change their behavior under (IA,DA). As a result, the truthful outcome prevails under (IA,DA), and school 1 never benefits from using IA when the other school does not use it. This asymmetry between school 1 and school 2 that school 1 is less likely to benefit from a stealing motive appears repeatedly in the paper.

\footnotetext{
${ }^{8}$ In this case, school 2's IA policy is still influential, but only for those poorer students. One can show that, in equilibrium, all type 1 students with $y_{1} \in\left(\left(s_{1}+s_{2}\right) / N,\left(s_{1}+s_{2}\right) / N+x\right)$ now make school 2 their top choice and will be admitted by the school in state 1 .
} 
Proposition 2 (unilateral incentive) Suppose that $\alpha_{1}=\alpha_{2}=0$ and that $y_{1}$ is uniformly distributed between $[0,1]$.

1. Suppose that $\pi_{2} u_{1}<u_{2}\left(\pi_{2} u_{1}>u_{2}\right)$. Under (DA,IA), (a) there does not exist a truthful equilibrium, and (b) there exists an equilibrium in which school 2's admitted applicants' average quality is improved (worsened) compared with under (DA,DA).

2. (IA,DA) and $(D A, D A)$ are outcome equivalent.

\section{Proof. Omitted.}

A few comments are in order here. First, given $\mu_{1}^{1}$ and starting from $\mu_{1}^{2}=\mu_{1}^{1}$, the number of applicants that are affected by school 2's IA increases as $\mu_{1}^{2}$ decreases. That is, starting with no demand uncertainty, an increase in the difference between $\mu_{1}^{1}$ and $\mu_{1}^{2}$ always increases the number of applicants whose behavior is affected. Second, whenever school 2 experiences an improvement of its average quality through using IA, school 1 experiences a worsening whereby. This is a straightforward result from the fact that the two schools have identical preferences for applicants. Third, some applicants are better off under (DA,IA) than under (DA,DA). Those type 1 applicants in region $\mathrm{B}$ now get into school 1 in state 2 under (DA,IA), while they can only get into school 2 under (DA,DA). ${ }^{9}$ Relatedly, we also note that these type 1 applicants have a higher expected utility compared with their slightly more qualified counterparts (with slightly lower $y_{1}$ ), who also adopt the same strategy. Somewhat ironically, just because the latter group is of better quality and is selected by school 2 - their top choice but less preferred school - the former group is able to get into their school 1.

\subsection{Competition}

We now study the equilibrium when both schools are allowed to pre-commit. We restrict our attention to equilibrium in which schools play only pure strategies. If $\pi_{2} u_{1}>u_{2}$, then it follows from the previous analysis that there are only two, outcome-equivalent, pure strategy equilibria, (DA,DA) and (IA,DA). The more interesting case is where $\pi_{2} u_{1}<u_{2}$, where school 2 strictly benefits from using IA provided that school 1 does not use it. In this case, we have

$$
A Q_{1}(I A, D A)=A Q_{1}(D A, D A)
$$

and

$$
A Q_{2}(D A, I A)<A Q_{2}(D A, D A) .
$$

\footnotetext{
${ }^{9}$ Thus, it is not the case that school 2's IA policy will be universally opposed by all applicants.
} 
$(\mathrm{IA}, \mathrm{DA})$ is an equilibrium if it is also true that

$$
A Q_{2}(I A, D A)<A Q_{2}(I A, I A)
$$

which means that, by using IA, school 1 can deter school 2 from using IA. We say that (IA,DA) is a preemptive equilibrium for school 1 if (3), (4) and (5) are satisfied. It is indeed the unique equilibrium (restricting to equilibria in which no dominated strategies are played) if it is also true that

$$
A Q_{1}(I A, I A) \leq A Q_{1}(D A, I A)
$$

otherwise, there will exist another pure strategy equilibrium: (DA,IA).

Assuming (3) and (4), whether a preemptive equilibrium exists depends on whether or not (5) holds, or whether or not $A Q_{2}(I A, I A)-A Q_{2}(I A, D A)>0$. Since (IA,DA) is outcome equivalent to (DA,DA), the difference can be decomposed into two components, in the following way:

$$
\begin{aligned}
& A Q_{2}(I A, I A)-A Q_{2}(I A, D A) \\
= & \left(A Q_{2}(I A, I A)-A Q_{2}(D A, I A)\right)+\left(A Q_{2}(D A, I A)-A Q_{2}(D A, D A)\right)
\end{aligned}
$$

where, according to (4), the second term in right-hand side is negative. To see when (7) is positive, it suffices to focus on the first term in the right-hand side, i.e., to study how school 2's average quality is changed from (DA,IA) to (IA,IA). Recall that under (DA,IA), in equilibrium, applicants with $y_{1}<\left(s_{1}+s_{2}\right) / N-x$ are admitted by the same school under both states; thus any new threat due to school 1's IA will not alter their behavior. However, it is not the case for applicants with $y_{1} \in T$ because they are admitted by different schools in different states. Now, they must choose between being admitted by school 2 in state 1 and not admitted by any school in state 2 , on the one hand, and being admitted by school 1 in state 2 and not admitted by any school in state 1 , on the other.

Since the respective utilities from the two choices are $\pi_{1} u_{2}$ and $\pi_{2} u_{1}$ for type 1 applicants and $\pi_{1} v_{2}$ and $\pi_{2} v_{1}$ for type 2 applicants, to understand these applicants' optimal strategies, we need to consider three cases. If $\pi_{1} u_{2}>\pi_{2} u_{1}$ (implying $\pi_{1} v_{2}>\pi_{2} v_{1}$ ), all applicants specify school 2 as their top choice. School 2's student quality under (IA,IA) is just the same as under (DA,IA). Substituting $A Q_{2}(I A, I A)-A Q_{2}(D A, I A)=0$ into (7), we find that (7) is negative and, as a result, there does not exist any preemptive equilibrium. If $u_{1} / u_{2}>\pi_{1} / \pi_{2}>v_{1} / v_{2}$, while those type 1 applicants will specify school 1 as their top choice, those type 2 applicants will specify school 2 as their top choice. Because of the pre-commitment, school 1 now need to admit less qualified applicants to replace the latter group. Thus a worsening relative to (DA,IA), or (6) is violated. Hence, (DA,IA) must be an equilibrium, whether or not any preemptive equilibrium exists. Finally, if $\pi_{1} v_{2}<\pi_{2} v_{1}$, 
all applicants will specify school 1 as their top choice. Then school 1 gets exactly the same applicants under (IA,IA) as under (DA,IA), and (6) is satisfied. Hence, if a preemptive equilibrium ever exists, it is also the unique equilibrium.

Proposition 3 (competition) Suppose that $\alpha_{1}=\alpha_{2}=0$ and that $y_{1}$ is uniformly distributed between $[0,1]$.

1. If $\pi_{2} u_{1}>u_{2}$, there are only two pure strategy equilibria: (DA,DA) and (IA,DA). They both lead to the same truthful outcome.

2. Suppose that $\pi_{2} u_{1}<u_{2}$.

(a) If $\pi_{1} u_{2}>\pi_{2} u_{1}$, there is a unique equilibrium of (DA,IA).

(b) If $u_{1} / u_{2}>\pi_{1} / \pi_{2}>v_{1} / v_{2}$, (DA,IA) is an equilibrium, and there may exist another equilibrium, (IA,DA).

(c) If $\pi_{1} v_{2}<\pi_{2} v_{1}$, there is a unique equilibrium: either (DA,IA) or (IA,DA).

\section{Proof. Omitted.}

To check if a preemptive equilibrium really exists, we first consider the scenario where $\pi_{1} v_{2}<\pi_{2} v_{1}$. Under (IA,IA), by specifying school 1 as their top choice, all applicants with $y_{1} \in T$ give up the opportunity to enter school 2 in state 1 , and school 2 must admit an equal number of applicants to replace them in this state. It is clear that applicants of both types with $y_{1} \in U \equiv\left(\left(s_{1}+s_{2}\right) / N,\left(s_{1}+s_{2}\right) / N+x\right)$ are replacements in equilibrium (highlighted as shaded region $\mathrm{C}$ in Figure 2).

The change in school 2's average quality can be represented as follows:

$$
\begin{aligned}
\Delta & \equiv A Q_{2}(I A, I A)-A Q_{2}(I A, D A) \\
& =\pi_{1}[n(C) m(C)-n(B) m(B)]+\pi_{2}[n(A) m(A)-n(B) m(B)],
\end{aligned}
$$

where $n($.$) and m($.$) are the number and the average quality of the applicants in the region$ concerned, and regions A, B, and C are depicted in Figure 2. Relative to (IA,DA), school 2's average quality worsens in state 1 and improves in state 2: in state 1, applicants in Region B are replaced by applicants in Region C; in state 2, applicants in Region B are replaced by applicants in Region A. If the state 1 effect dominates, there will be a worsening of school 2's overall average quality. In other words, if $\Delta>0$, a preemptive equilibrium exists. The analysis is simplified by the fact that the number of applicants, as well as their average quality, in each region is independent of $\pi_{1}$ and $\pi_{2}$.

Proposition 4 Suppose that $u_{2}>\pi_{2} u_{1}$ and $\pi_{1} v_{2}<\pi_{2} v_{1}$. Then there exist parameter values such that $\Delta>0$ (i.e., a preemptive equilibrium exists) if and only if $s_{2} / s_{1}<2 \pi_{1} / \pi_{2}$. 

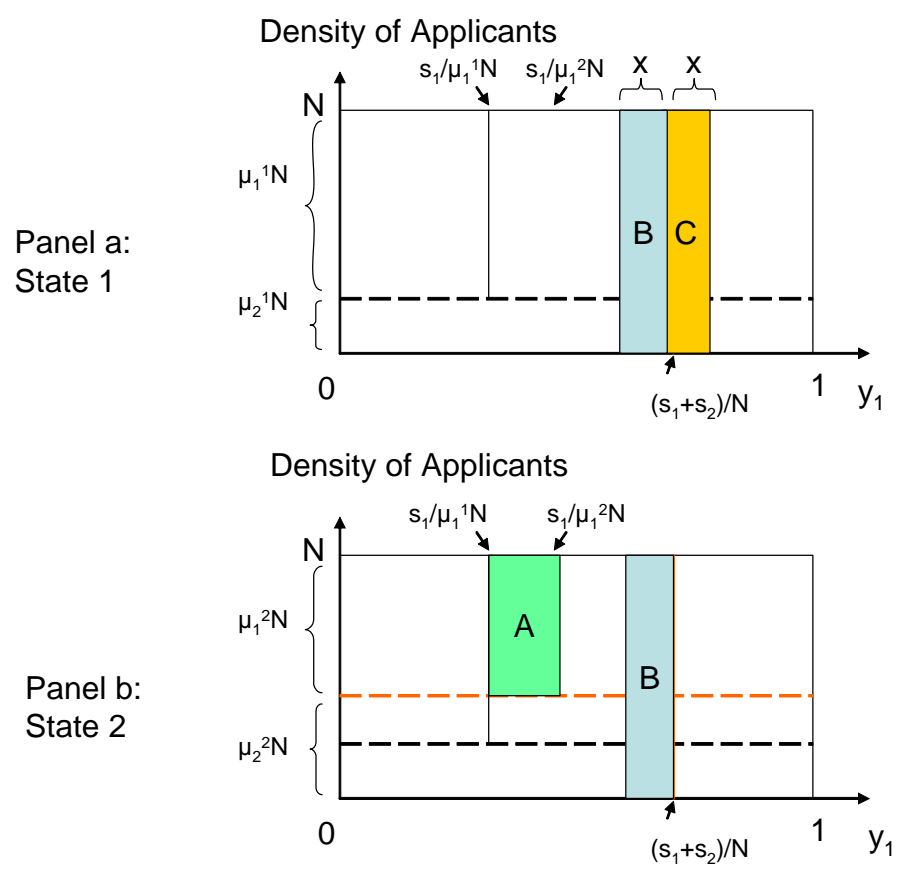

Figure 2: The shaded regions show the change in admitted students that school 2 will encounter when the policy changes from (IA,DA) to (IA,IA).

There are two ways to understand the condition $s_{2} / s_{1}<2 \pi_{1} / \pi_{2}$. First, given that the other parameters are unchanged and given $s_{1}$, the greater $s_{2}$, the less likely that a preemptive equilibrium exists. Comparing (IA,IA) with (IA,DA), we find that school 2's loss in state 1 is invariant to $s_{2}$ but its gain in state 2 is increasing in $s_{2}$. Hence, when $s_{2}$ is sufficiently large, IA is so attractive to school 2 that its use is never thwarted. Second, $\pi_{1} / \pi_{2}$ has to be sufficiently large for $\Delta>0$. The reason is that the greater the likelihood of state 1 , the greater weight we put on state 1's effect, which is favorable for $\Delta>0$. To get more concrete ideas, suppose that $u_{2} / u_{1}=v_{1} / v_{2}=0.8$. Then, there always exist parameters of a preemptive equilibrium when $s_{2} / s_{1}<1.6$ and never exist when $s_{2} / s_{1}>2.5$.

We next study the scenario when $u_{1} / u_{2}>\pi_{1} / \pi_{2}>v_{1} / v_{2}$ and check if a preemptive equilibrium really exists. The equilibrium student intakes for school 2 under (IA,IA) are depicted as shaded regions in Figure $3 .^{10}$ Note that, in state 1, those in region $\mathrm{C}$ are admitted to replace those type 1 applicants in Region B' who, with school 1 as their top

\footnotetext{
${ }^{10}$ As depicted, Region $\mathrm{C}$ consists of both type 1 and type 2 applicants. It is, however, possible, that it consists solely of type 2 applicants. This is the case when applicants in Region B' is not too numerous so that they can be completely replaced by type 2 applicants.
} 

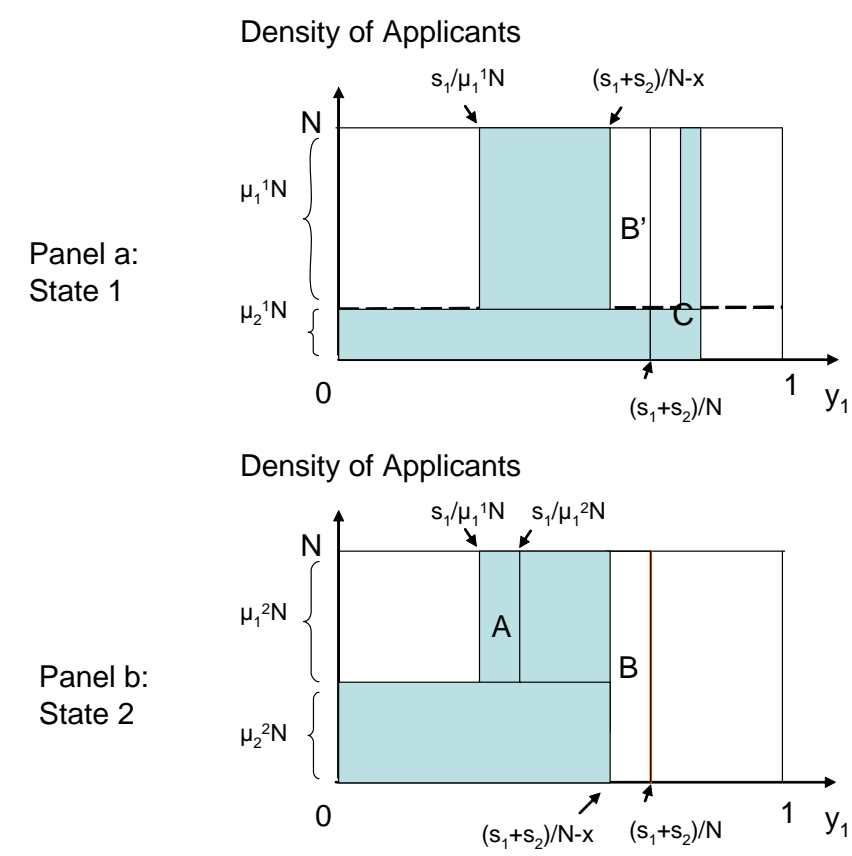

Figure 3: The shaded regions show that school 2 admits in equilibrium under (IA,IA). Region B' refers to those type 1 applicants with $y_{1} \in T$.

choice, are not admittable. Also note that in state 2 school 2 admits exactly the same applicants as under (DA,IA). The change in average quality for school 2 can be written as

$$
\begin{aligned}
\Gamma & \equiv A Q_{2}(I A, I A)-A Q_{2}(I A, D A) \\
& =\pi_{1}\left[n(C) m(C)-n\left(B^{\prime}\right) m\left(B^{\prime}\right)\right]+\pi_{2}[n(A) m(A)-n(B) m(B)],
\end{aligned}
$$

where the first term on the right-hand side is positive and the second term is negative. If the first term dominates, then the overall student quality is worsened. Again, the analysis is simplified by the fact that in each region the number of applicants, as well as the corresponding average quality, is invariant to $\pi_{1}$ and $\pi_{2}$.

Proposition 5 Suppose that $u_{2}>\pi_{2} u_{1}$ and $u_{1} / u_{2}>\pi_{1} / \pi_{2}>v_{1} / v_{2}$. Then there exist parameter values such that $\Gamma>0$ (a preemptive equilibrium exists) if

$$
\frac{s_{2}}{s_{1}}<\frac{\mu_{1}^{1}}{1-\mu_{1}^{1}} \frac{\pi_{1}}{\pi_{2}}
$$

Here is an example. Suppose that $s_{1}=s_{2}=0.25, N=1, \mu_{1}^{1}=1, \mu_{1}^{2}=0.6$. Then 
any $v_{1}, v_{2}, u_{1}, u_{2}$ satisfying $0.6>u_{2} /\left(u_{1}+u_{2}\right)$ will ensure a preemptive equilibrium. The intuition behind the roles of $s_{2} / s_{1}$ and of $\pi_{1} / \pi_{2}$ is analogous to that in Proposition 4 and is not repeated. Note that unlike in Proposition 4, (10) is only a sufficient condition, rather than a sufficient and necessary condition. Thus violation of (10) does not necessarily mean the non-existence of preemptive equilibrium.

\subsection{Summary}

We have identified two motives for using IA. A school may use it to steal applicants who otherwise would not put it as their top choice. We call this the stealing motive. Being able to influence applicants' behavior, however, need not imply an improvement of the school's average quality: IA can be influential yet self-defeating. While the popular school does not have the stealing motive, the less popular school does so long as it is not too inferior to the popular school from applicants' point of view. We have also identified the preemptive motive, whereby the popular school uses IA to thwart the less popular school from adopting its own IA. In particular, a preemptive equilibrium exists if $s_{2} / s_{1}$ is small enough. On the contrary, when $s_{2} / s_{1}$ is sufficiently large, provided that $u_{2} / u_{1}$ is not too low, the less popular school has IA as its dominant strategy.

\section{Demand Uncertainty with Heterogeneous School Pref- erences $\left(\alpha_{1} \neq \alpha_{2}\right)$}

We argue here that the insights discovered - that IA being influential is different from its being advantageous, that there is asymmetry between the two schools regarding the stealing motive, and that school 1 may have the preemptive motive -remain true when school preferences are heterogeneous. As in the previous case, diagrammatic analysis proves to be very useful. But in this case, the diagram we use is somewhat different, because both $y_{1}$ and $y_{2}$ are relevant and should be shown.

\subsection{Benchmark}

Under (DA,DA), the truthful outcome is characterized by a cutoff standard for each state set by each school so that every applicant with a better attribute pair will be admitted provided that she writes it as her top choice or, if not, she has been declined by her top choice school. Referring to Figure 4, we use $L L_{i}, M M_{i}$ to denote the cutoff lines set by school 1 and school 2 under state $i=1,2$. There are three different possibilities regarding the relative positions of these four lines. In Panel a, school 1 is so popular that, in the truthful equilibrium, it never admits any type 2 applicant; as a result, school 2's cutoffs are the same under both states - as in the homogeneous school preferences case studied in the 
last Section. In Panel b, school 1 admits type 2 applicants only under state 2; in Panel c, school 1 admits type 2 applicants under both states.

For $i, j=1,2$ and $i \neq j$, school $i$ is said to be (i) dominant if in the truthful outcome it never admits a type $j$ applicant; (ii) semi-dominant if it admits some type $j$ applicants under only one state, and (iii) non-dominant if it admits some type $j$ applicants under both states. Clearly, school 1 is, respectively, dominant, semi-dominant, and non-dominant in the first three panels depicted in Figure 4. It is also clear that school 2 cannot be dominant and only school 1 can be. Moreover, given assumption (2), school 1 is always dominant if $\alpha_{1}=\alpha_{2}$. One can show that, given $\alpha_{1}$, there exist $\bar{\alpha}$ and $\underline{\alpha}$, where $\bar{\alpha}>\underline{\alpha}>\alpha_{1}$, so that school 1 is dominant if $\alpha_{2} \in\left[\alpha_{1}, \underline{\alpha}\right)$, semi-dominant if $\alpha_{2} \in(\underline{\alpha}, \bar{\alpha})$, and non-dominant if $\alpha_{2} \in(\bar{\alpha}, \infty)$.

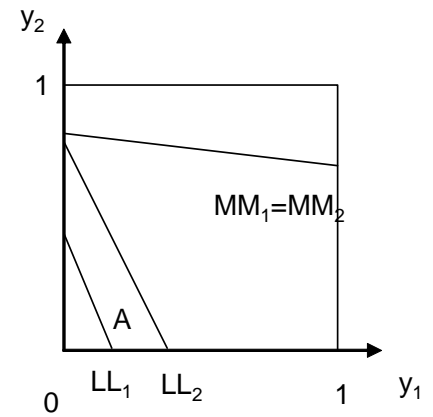

Panel a: school 1 is dominant

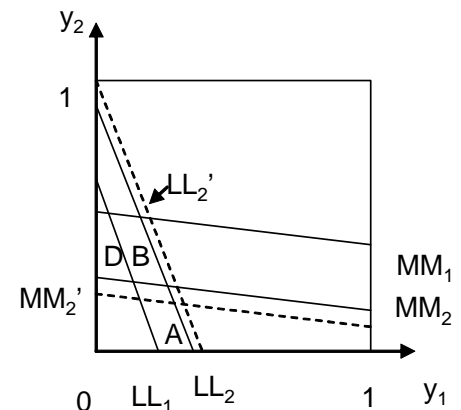

Panel c: school 1 is non-dominant

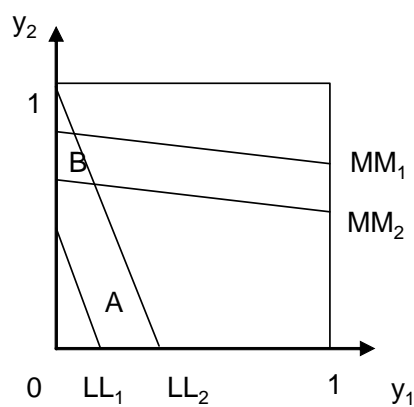

Panel b: school 1 is semi-dominant

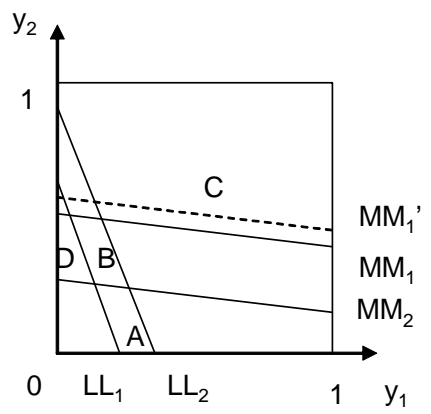

Panel d: school 1 is non-dominant

Figure 4: $L L_{1}$ and $L L_{2}\left(M M_{1}\right.$ and $\left.M M_{2}\right)$ are cutoffs set by school 1 (2) in the two states under (DA,DA). Corresponding lines with asterisks are cutoffs under (DA,IA). 


\subsection{Unilateral Incentive}

The following Proposition, which is closely related to Proposition 2, can be obtained.

Proposition 6 Suppose that $\alpha_{1} \neq \alpha_{2}$ and $\left(y_{1}, y_{2}\right)$ is uniformly distributed in $[0,1] \times[0,1]$.

1. Suppose that $\pi_{2} u_{1}<u_{2}\left(\pi_{2} u_{1}>u_{2}\right)$. Under (DA,IA), (a) there does not exist a truthful equilibrium, and (b) there exists an equilibrium in which school 2's average quality is improved (worsened) compared with under (DA,DA).

2. (IA,DA) and $(D A, D A)$ are outcome equivalent if school 1 is dominant or semi-dominant.

3. If school 1 is non-dominant and $\pi_{1} v_{2}<v_{1}\left(\pi_{1} v_{2}>v_{1}\right)$, then under (IA,DA) (a) there does not exist a truthful equilibrium, and (b) there exists an equilibrium in which school 1 's average quality is improved (worsened) compared with under (DA,DA).

Proof. Omitted.

To understand result 1 , recall from the previous section that the purpose of using IA for school 2 is to steal those type 1 applicants who would otherwise enter different schools in different states under (DA,DA). Exactly the same insight holds true here. Type 1 applicants with attribute pairs in Region $\mathrm{A}$ in Panels a, b and c of Figure 4 are such applicants. If $\pi_{2} u_{1}>u_{2}$, they do not deviate and school 2 suffers for having to replace them, in state 1 , with poorer quality applicants that are not admitted under (DA,DA). ${ }^{11}$ If $\pi_{2} u_{1}<u_{2}$, on the other hand, they are just as willing to deviate from truthful reporting as are their counterparts under homogeneous preferences, rendering the truthful equilibrium infeasible.

We use the case of the non-dominant school 1 (Panel c of Figure 4) to illustrate the equilibrium under (DA,IA); the analyses for the cases of dominance and semi-dominance are similar. In state 1 , the cutoff lines remain to be $L L_{1}$ and $M M_{1}$ as under (DA,DA); in state 2, school 2's cutoff line shifts down to $M M_{2}^{\prime}$ while school 1's cutoff line shifts right to $L L_{2}^{\prime}$. Now that school 2 is able to admit good type 1 applicants that it otherwise does not admit, its cutoff value is made more stringent, while school 1's cutoff is relaxed. ${ }^{12}$

Now we turn to the second point. The argument is exactly the same as under the homogeneous school preferences case. When school 1 is dominant or semi-dominant, there does not exist a group of type 2 applicants who enter different schools in different states under (DA,DA). As a result, school 1 cannot gain from using IA. When school 1 is nondominant (the third point), such a group does exist (region D in Panel c in Figure 4). Then school 1 can break the truthful equilibrium by using IA and will indeed benefit from doing so through the stealing effect when $\pi_{1} v_{2}<v_{1}$. Since the more apart $\alpha_{1}$ and $\alpha_{2}$ are the more

\footnotetext{
${ }^{11}$ As a result, school 2's cutoff in state 1 will shift, say, to MM1' in panel d of Figure 4.

${ }^{12}$ This outcome is supported by the following equilibrium strategies: (1) all type 1 applicants in the region bounded by $L L_{1}, M M_{1}, L L_{2}$ and the horizontal axis specify school 2 as their top choice, and (2) all type 1 applicants on the right-hand side of $L L_{1}$, as well as all type 2 applicants, report truthfully (other applicants' strategies are inconsequential).
} 
likely school 1 is non-dominant, this suggests a subtle role played by school preferences divergence that determines if school 1 has the stealing effect.

A remark is in order here. Unlike in the homogeneous school preferences case, it is no longer the case that whenever one school benefits from using IA, the other school will suffer as a consequence. Applicants whom one school thinks not very good may now be considered by the other as very good. Thus, the school that does not use the IA policy may admit good applicants that it otherwise does not admit under (DA,DA). If this effect is significant enough, the school may be made better off as well.

\subsection{Competition}

The previous subsection demonstrated identical results as under homogeneous school preferences so long as school 1 is dominant or semi-dominant. This means that Proposition 3 continues to hold under heterogeneous school preferences so long as school 1 is dominant or semi-dominant. Since a preemptive equilibrium presumably exists under some conditions, we do not belabor to venture into the exact conditions here.

A new research question here is what the equilibrium is like when school 1 is nondominant and, in particular, when both schools each have an incentive to use IA given that the other school does not use IA (i.e., $\pi_{1} v_{2}<v_{1}$ and $\pi_{2} u_{1}<u_{2}$ ). Our simulation studies suggest that both schools using the IA policy is an equilibrium outcome in ample environments. In the simulation, we assume that $u_{2} / u_{1}=v_{1} / v_{2}=0.8, s_{1}=s_{2}=0.25, N=$ $1, \alpha_{1}=0, \mu_{1}^{1}=0.8$. With these parameters, the necessary and sufficient condition for $\pi_{1} v_{2}<$ $v_{1}$, and $\pi_{2} u_{1}<u_{2}$ is $\pi_{1} \in(0.2,0.8)$. Two parameter values of $\alpha_{2}$ are used here, $\alpha_{2}=1$, and $\alpha_{2}=2$, which are verified to ensure the non-dominance of school 1 . We attempt on two specifications for the distributions of $\left(y_{1}, y_{2}\right)$ : uniform distribution and a cumulative function with associated density function $g\left(y_{1}, y_{2}\right)=1+\left(2 y_{1}-1\right)\left(2 y_{2}-1\right)$.

Figure 5 reports the results in terms of parameter values of $\pi_{1}$ and $\mu_{1}^{2} \cdot{ }^{13}$ Both schools using IA is an equilibrium outcome for parameter values above, respectively, the $C C$ curves when density function $g($.$) is used and the N N$ curves when a uniform distribution is used. For the case of $\alpha_{2}=2$, both using IA is an equilibrium outcome for all parameter values of $\pi_{1}$ and $\mu_{1}^{2}$ when $g($.$) is used and hence no N N$ curve is depicted. Some interesting comparative statics are obtained: for both distributions, it is more likely for the outcome to be viable when the divergence of school preferences is greater and when $\mu_{1}^{2}$ is large. The first result is consistent with the partial mutual exchange of applicants pointed out in the last subsection, while the insight into the second result is not straightforward. The bottom line is clear though: there are ample scenarios in which both schools using IA can be an equilibrium outcome.

\footnotetext{
${ }^{13}$ Though (2) implies that $\mu_{1}^{2} \geq 0.5$, our analysis in fact applies for $\mu_{1}^{2}<0.5$. We report a larger range of $\mu_{1}^{2}$ here.
} 

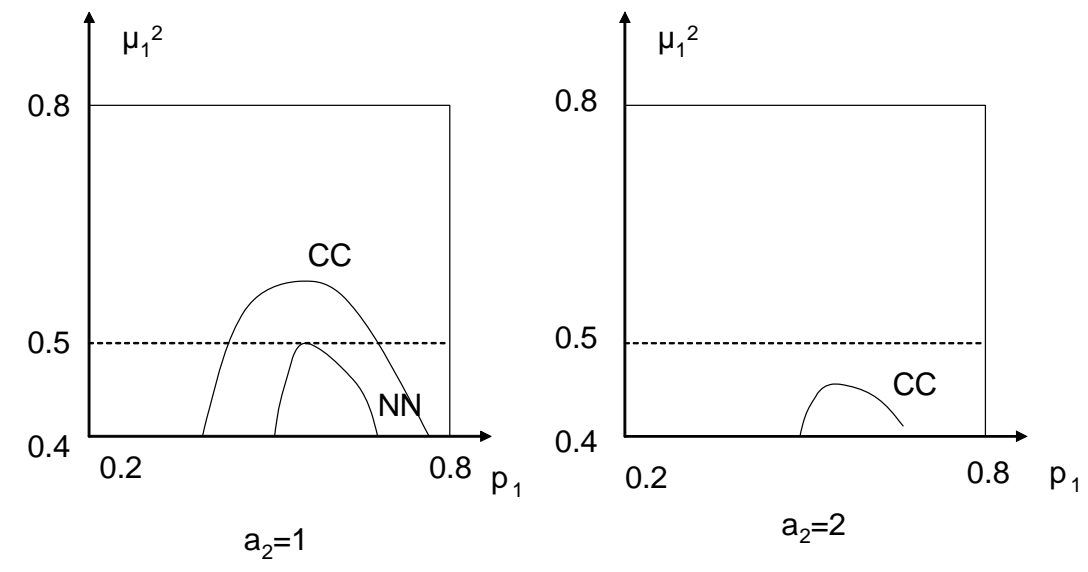

Figure 5: Both schools using IA is an equilibrium if and only if $\mu_{1}^{2}$ and $\pi_{1}$ are in the respective region outside the CC curve (when $y_{1}$ and $y_{2}$ are positively correlated) and NN curve (when $y_{1}$ and $y_{2}$ are uncorrelated).

\section{$5 \quad$ Ranking Uncertainty}

Here we study an alternative setting in which applicants are not informed of their attributes or relative ranking. When applicants are required to specify their preferences before taking their public exams (the scores of which will become their attributes), then there will be information asymmetry between applicants and schools, which will make admission decisions only after knowing the exam results. This is the case for several provinces in the Chinese national college admissions system. ${ }^{14}$

We argue that this relative ranking uncertainty may also lead to strategic use of the IA policy and yield similar insights regarding the stealing motive and the preemptive motive. To make the point, we focus on a very simple version. Like in the previous model, there are two schools with seats, $s_{1}$ and $s_{2}$, for which a mass of applicants of size $N$ are competing. The two schools' preferences are represented by $\alpha_{1}=0$ and $\alpha_{2} \geq \alpha_{1}$. Each applicant is

\footnotetext{
${ }^{14}$ Each Chinese province runs its own admissions system for its resident students. Some provinces require students to apply before they take the national examination; some require students to apply after they take the exam while before they learn of their scores, the other require students to apply even after scores are known. It should be noted that, since students in each province are competing only among themselves to enter colleges with pre-determined places allotted to that province, they do not compete with students of another province.
} 
characterized by an attribute pair $\left(y_{1}, y_{2}\right)$ uniformly distributed over $[0,1] \times[0,1]$. The distribution function is commonly known, and schools know each applicant's attribute pair when evaluating applicants. Despite this, each applicant does not know her own attribute pair (nor the attribute pair of any other applicant); in other words, each applicant is ex ante identical. This is the quintessential case of ranking uncertainty.

To focus on the role of ranking uncertainty, we also assume that applicants have identical preferences. For each applicant, the utility of attending school $i$ is $u_{i}, i=1,2$, so that $u_{1}>u_{2}$; the utility of not attending any school is zero. Occasionally, we will call school 1 the popular school and school 2 the less popular school. (Hence, there do not exist two separate states of the world regarding demand. This assumption allows us to focus solely on the role of ranking uncertainty.)

\subsection{Identical School Preferences $\left(\alpha_{1}=\alpha_{2}\right)$}

\subsubsection{Unilateral Incentive}

We first study the case of identical school preferences, i.e., $\alpha_{1}=\alpha_{2}=0$, and as such we can represent each applicant's attribute simply by her first attribute, $y_{1}$. Under (DA,DA) the equilibrium is such that all applicants make school 1 their top choice. It follows immediately that school 1 never has the stealing motive. It is not be the case for school 2, however. Suppose under (DA,IA) all applicants behave truthfully. Then each applicant can benefit from deviating to make school 2 her top choice so long as $u_{2} / u_{1}$ is sufficiently high, and the truthful equilibrium is infeasible. This means that in equilibrium a number of applicants will specify school 2 as their top choice, and if this number (denoted by $b$ ) is large enough, school 2 will indeed benefit from the IA policy. The equilibria under (DA,DA) and (DA,IA) are shown in Figure 6, where $a$ and $b$, respectively, is the corresponding number of applicants who specify school 1 and school 2 as their top choice.

Proposition 7 (Unilateral incentive) Suppose that $\alpha_{1}=\alpha_{2}=0$ and that $y_{1}$ is distributed uniformly over the [0,1] interval.

1. $(I A, D A)$ and $(D A, D A)$ are outcome equivalent.

2. Under (DA,IA), if $u_{2} / u_{1}<s_{1} /\left(N-s_{2}\right)$, there exists a truthful equilibrium.

3. Under (DA,IA), if $u_{2} / u_{1}>s_{1} /\left(N-s_{2}\right)$, then (a) there does not exist a truthful equilibrium, but (b) there exists an equilibrium in which $b \in\left(s_{2}, N\right)$ and in this equilibrium $A Q_{2}(D A, I A)<A Q_{2}(D A, D A)$ if and only if $u_{2} / u_{1}>1 / 2$.

According to point 3, the condition for school 2's IA to influence applicants' behavior is different from the condition for it to benefit from the policy. For instance, consider the parameter values $s_{1}=s_{2}=N / 4$ and $u_{2} / u_{1}=0.4$. Then school 1's IA is able to influence 


\section{Density of students}

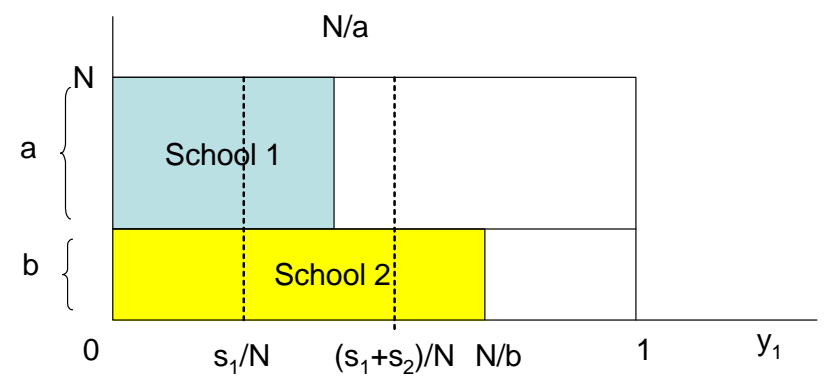

Figure 6: The two dotted lines show the cutoffs under (DA,DA). The two shaded regions show the admitted students under (DA,IA).

applicants' behavior but its average applicant quality will worsen. More generally, provided that $2 s_{1}+s_{2}<N$, there exists a nonvanishing range of $u_{2} / u_{1} \in\left(s_{1} /\left(N-s_{2}\right), 1 / 2\right)$ such that school 2's IA is influential yet self-defeating.

\subsubsection{Competition}

Given that $u_{2} / u_{1}>s_{1} /\left(N-s_{2}\right)$, the equilibrium under (DA,IA) has the following simple but powerful feature: relative to school 2, school 1's cutoff value must be more stringent. (Or else, each applicant would strictly prefer to making school 1 as their top choice, violating the fact that school 1's cutoff value is less stringent.) This implies that school 1 admits only among those who specify it as their top choice. Taking into account the property that $b>s_{2}$ (Part 3 of Proposition 7), we reckon that neither school will admit any applicant not specifying it as her top choice. In other words, the outcome under (DA,IA) is exactly the same as that under (IA,IA). Noting the outcome equivalence of (DA,DA) and (IA,DA), we conclude that, regardless of school 2's policy choice, school 1 cannot worsen school 2 through using IA. School 2's IA is nonpreemptive.

Proposition 8 (Competition) Suppose that $\alpha_{1}=\alpha_{2}=0$ and that $y_{1}$ is distributed uniformly over the [0,1] interval.

1. If $u_{2} / u_{1}>\max \left\{s_{1} /\left(N-s_{2}\right), 1 / 2\right\}$, then there is a unique equilibrium outcome, which is the same as under $(D A, I A)$. 
2. If $u_{2} / u_{1}<\max \left\{s_{1} /\left(N-s_{2}\right), 1 / 2\right\}$, then there is a unique equilibrium outcome, which is the same as under $(D A, D A)$.

\section{Proof. Omitted.}

According to the first result, when $u_{2} / u_{1}$ is high enough, school 2 will gain from using IA, and its use of IA is not deterred by school 1. In fact, school 2's student intake is invariant to whether or not school 1 adopts IA. There are two pure strategy equilibria: (DA,IA) and (IA,IA) and they are outcome equivalent. According to the second point, when $u_{2} / u_{1}$ is not high enough, school 2 will not gain from using IA and will not use it. There are two pure strategy equilibria: (DA,DA) and (IA,DA) and they are outcome equivalent. To conclude, we have found not only an asymmetry between the two schools regarding the stealing effect but also the lack of preemptive ability on the part of the popular school. ${ }^{15}$

\subsubsection{More Information}

Despite our focus on the quintessential case, we think that the general insights will still hold when applicants have more information about the rankings among themselves. We illustrate this with a simple variant. We continue to assume that the two schools care about $y_{1}$ only. Assume that each applicant knows whether her $y_{1}$ is of grade A or grade B and that grade A is a better grade. Consider the case of identical school preferences and assume that among $N$ applicants, $e N$ of them belong to grade A applicants and they know it; the rest belong to grade B applicants and they know it too. Suppose that $e N<s_{1}$.

Under (DA,DA), applicants still have reporting truthfully as their equilibrium. Under (DA,IA), grade A applicants are certain that they can enter the same school (school 1), while Grade B applicants are not. In this case, if $u_{2} / u_{1}$ is sufficiently high, grade B applicants will have unilateral incentives to deviate from the truthful equilibrium. More precisely, we can show that if $u_{2} / u_{1}>\left(s_{1}-e N\right) /\left(N-s_{2}\right)$, the truthful equilibrium is no longer viable; instead, a number of grade B applicants $\left(b>s_{2}\right)$ will make school 2 their top choice. We can show that $A Q_{2}(D A, I A)<A Q_{2}(D A, D A)$ provided that $u_{2} / u_{1}>1 / 2$. To say the least, though the applicants know more about themselves, school 2 can still influence the applicants' behavior through and benefit from using IA. ${ }^{16}$

\footnotetext{
${ }^{15}$ Here we assume that applicants' uncertainty about the ranking among them is because of an information asymmetry between schools and applicants. We can also think of scenarios in which there is no information asymmetry associated with applicants' ranking uncertainty. For instance, in many other places, notably commonwealth countries in which an applicant's public examination result in each subject is normally summarized by a letter grade (or sub-grade). Uncertain about how schools will break ties among students with the same grade, applicants are also facing a sort of relative ranking uncertainty. (See Erdil and Ergin (forthcoming) and Abdulkadiroglu, Pathak and Roth (2006) on the role of the tie-breaking rule.) As far as the effect of IA on applicants' behavior, our results obtained here apply. We cannot, however, be as assertive regarding the benefits of using IA to the school.

${ }^{16}$ Likewise, we can show that school 1 does not have an incentive to use IA provided that school 2 does not use IA, and that school 2's optimal policy choice is independent of what school 1 will do when both schools are allowed to pre-commit.
} 


\subsection{Heterogeneous School Preferences}

Despite its absence under homogeneous preferences, a preemptive equilibrium by school 1 may exist if school preferences are sufficiently heterogeneous. To see this, we assume that $\alpha_{1}=0$ and $\alpha_{2}=\infty$ such that school 1 cares about the applicants' first attribute, $y_{1}$, and school 2 cares only about their second attribute, $y_{2}$. This is the most extreme case of heterogeneous preferences. Under (DA,DA), applicants still report truthfully, and school 1 admits applicants whose $y_{1} \leq c_{1}=s_{1} / N$ and school 2 admits applicants whose $\left(y_{1}, y_{2}\right)$ satisfies $y_{1}>c_{1}$ and $y_{2} \leq c_{2}=s_{2} /\left(N-s_{1}\right)$, where $c_{1}$ and $c_{2}$ are the two cutoffs on $y_{1}$ and $y_{2}$ that the two schools, respectively, set in equilibrium. We have the following proposition regarding the unilateral incentive to use IA.

Proposition 9 Suppose the $\alpha_{1}=0, \alpha_{2}=\infty$, and $\left(y_{1}, y_{2}\right)$ is distributed uniformly over the [0,1] square.

1. $(I A, D A)$ and $(D A, D A)$ are outcome equivalent.

2. Under (DA,IA), if $u_{2} / u_{1}<s_{1} /\left(N-s_{2}\right)$, there exists a truthful equilibrium; if $u_{2} / u_{1}>$ $s_{1} /\left(N-s_{2}\right)$, then (a) there does not exist a truthful equilibrium, but (b) there exists a unique equilibrium, in which $b=N$ and $A Q_{2}(D A, I A)<A Q_{2}(D A, D A)$.

The first result is derived from the fact that school 1 has no incentive to use IA given that school 2 does not use it. The bandwagon phenomenon in result 2.b is a joint outcome of school 1's DA and the two schools' orthogonal preferences. Because of school 1's DA, those applicants who did not specify school 1 as their top choice are not discriminated against by school 1 when they become available for its selection. Because of the schools' orthogonal preferences, these applicants are viewed by school 1 as good as those who specified school 1 as their top choice. As a result, they will be admitted with the same probability by school 1 as if they chose it as their top choice. Foreseeing this, they find it optimal to make school 2 their top choice provided that $u_{2}$ is sufficiently high. In the identical school preferences case, on the contrary, those specifying school 2 as their top choice and subsequently declined by school 2 are of inferior quality from school 1's point of view and will not be admitted. This difference leads to a very significant difference in terms of the applicants' behavior.

Given that $b=N$, school 2 has all the applicants to select from, and it is not surprising to see that the average quality of its students will improve. We note in passing that under (DA,IA) the two schools will have cutoffs $c_{1}=s_{1} /\left(1-c_{2}\right) N$ and $c_{2}=s_{2} / N$, with average qualities $A Q_{1}(D A, I A)=s_{1} / 2\left(1-s_{2}\right)$ and $A Q_{2}(D A, I A)=s_{2} / 2 N$. Compared with their counterparts under (DA,DA), we know that school 1 is worse off while school 2 is better off. Therefore, the ability to influence applicants' behavior is equivalent to gaining from the IA policy. 
We next note that, if $u_{2} / u_{1}>s_{1} /\left(N-s_{2}\right)$ and both schools are allowed to pre-commit to IA, there are two asymmetric equilibria: (IA,DA) and (DA,IA). The policy pair (IA,IA) is not an equilibrium because given that the other school uses IA, each school finds it optimal not to use it, i.e., we have $A Q_{1}(D A, I A)<A Q_{1}(I A, I A)$ and $A Q_{2}(I A, D A)<A Q_{2}(I A, I A)$.

Proposition 10 Suppose that $\alpha_{1}=0, \alpha_{2}=\infty,\left(y_{1}, y_{2}\right)$ is distributed uniformly over the [0,1] square, and $u_{2} / u_{1}<s_{1} /\left(N-s_{2}\right)$. There are two pure strategy equilibria: (IA,DA) and $(D A, I A)$.

(IA,DA) is a preemptive equilibrium. Not directly benefiting from the use of IA, school 1 can deter school 2 from using IA. Thus, the asymmetry between the two schools that we have seen under homogenous preferences is somewhat diluted.

\subsection{Summary}

As in the demand uncertainty model, we have found in this ranking certainty model that there is a difference between an influential IA policy and a beneficial IA policy, and that there is an asymmetry between the two schools regarding the stealing motive. Regarding the preemptive motive, new results have arisen. There is little the popular school can do to prevent the less popular school from choosing IA when the two schools have like preferences. When they are sufficiently different, however, the popular school can successfully use IA as a defensive policy.

\section{Concluding Remarks}

Misrepresenting preferences on the part of proposers has been documented and analyzed in admissions schemes, such as the Boston mechanism, in which preferential treatment is exogenously given. The Gale-Shapley mechanism, however, is acclaimed for its immunity to this problem. Taken the students-propose-to-schools variant for illustration, the literature has established that, students still have the incentive to report their preferences truthfully despite manipulation on the part of schools. As far as we are aware, our paper is the first to point out this is not generally true: within the Gale-Shapley framework, there still exists manipulation on the part of schools that leads to misreporting on the part of students. We think that pointing out this on its own is a contribution of the paper.

Two natural questions arise from this study. What can we do to reduce the scopes and effects of endogenous preferential treatment? Should endogenous preferential treatment be banned? Regarding the first question, since immediate acceptance policy has bites only when applicants face uncertainty, one natural way to mitigate the problem is to reduce the scope of uncertainty. Demand uncertainty can be reduced by a more thorough survey on applicants' preferences before application, while ranking uncertainty can be reduced 
by providing finer exam grades and scores (in the case of commonwealth countries) or postponing the application period until applicants know their exam scores (in the case of several Chinese provinces). Moreover, since public availability of past admissions records strengthens schools' long run reputational concerns, the education agency can judiciously reduce the release of such records for public access. In this case, schools' pre-commitments are discredited and have no bites provided that applicants no longer believe in them.

The second question is more difficult to answer. In our model, we assumed that schools adopted preferential treatment simply out of a strategic consideration, and the motivational consideration was completely neglected. When students' motivations and commitments are important to success in their study, schools naturally want to admit students who like them most, other things being equal. ${ }^{17}$ If a school adopts preferential treatment out of this consideration, there is nothing wrong with it and banning preferential treatment does not seem justifiable. To answer the question satisfactorily, therefore, it requires studying a model that also takes into account the motivational consideration. We hope that this issue can be tackled in future studies.

Dissatisfied with the centralized admissions system, either because of a strategic consideration or because of a motivational consideration, schools may opt to use alternative channels, such as early admissions or admissions that are run parallel to centralized admissions. We think that the interactions between centralized admissions and alternative admissions channels are an issue warranted future studies. The literature of college admissions and school choice can then be linked to that of early contracting (see, e.g., Li and Rosen, 1998; Roth and Xing, 1994; and Suen, 2000).

\footnotetext{
${ }^{17}$ The emphasis of one's motivation in one's performance and its impact on the design of organizations have received increasing attention lately (see, e.g., Hart and Moore 2002, and Besley and Ghatak 2005). This line of studies suggests that the motivational consideration pointed out here is worth further investigation.
} 


\section{References}

[1] Abdulkadiroğlu, Atila and Tayfun Sőnmez (2003), "School Choice: A Mechanism Design Approach," American Economic Review, 93(3): 729-748.

[2] Abdulkadiroğlu, Atila, Parag A. Pathak and Alvin E. Roth (2006), "Strategy-proofness versus Efficiency in Matching with Indifferences: Redesigning the NYC High School Match," mimeo, Duke University.

[3] Abdulkadiroğlu, Atila, Parag A. Pathak, Alvin E. Roth, and Tayfun Sőnmez (2005), "The Boston Public School Match," American Economic Review, Papers and Proceedings, May 2005.

[4] Abdulkadiroğlu, Atila, Parag A. Pathak, Alvin E. Roth, and Tayfun Sőnmez (2006), "Changing the Boston Mechanism: Strategyproofness as Equal Access," Unpublished mimeo, Harvard University.

[5] Besley, Timonthy and Maitreesh Ghatak (2005), "Competition and Incentives with Motivated Agents," American Economic Review, 616-636.

[6] Erdil, Aytek and Haluk Ergin (forthcoming), "What's the Matter with Tie-breaking? Improving Efficiency in School Choice," American Economic Review.

[7] Ergin, Haluk and Tayfun Sőnmez (2006), "Games of Social Choice under the Boston Mechanism," Journal of Public Economics, 90: 215-237.

[8] Gale, David and Lloyd S. Shapley (1962), "College Admissions and the Stability of Marriage," American Mathematical Monthly, 69(1): 9-15.

[9] Hart, Oliver and Bengt Holmström (2002), "Vision and Scope of the Firm," mimeo, 2002.

[10] Roth, Alvin E. (1989), "Two-sided matching with incomplete information about others' preferences," Games and Economic Behavior, 1:191-209.

[11] Roth, Alvin E and Marilda A. O. Sotomayor (1990), Two-sided matching: A study in game theoretic modeling and analysis. New York: Cambridge University Press, 1990.

[12] Roth, Alvin E and Elliot Peranson (1999), "The Redesign of the Matching Market for American Physicians: Some Engineering Aspects of Economic Design." American Economic Review, 89(4): 748-80.

[13] Roth, Alvin E. and Xiaolin Xing (1994) "Jumping the Gun: Imperfections and Institutions Related to the Timing of Market Transactions," American Economic Review, 84(4): 992-1044 
[14] Sonmez, Tayfun (1997) "Manipulation via Capacities in Two-Sided Matching Markets" Journal of Economic Theory, 77(1): 197-204

[15] Roth, Alvin E. Uriel G. Rothblum (1984), The Evolution of Labor Market for medical Interns and Residents: A Case Study in Game Theory," Journal of Political Economy 92(6): 991-1016.

[16] Li, Hao and Sherwin Rosen (1998) "Unraveling in Matching Markets," American Economic Review, 88(3):371-387.

[17] Suen, Wing (2000) "A Competitive Theory of Equilibrium and Disequilibrium Unraveling in Two-Sided Matching," RAND Journal of Economics, 31:101-120. 


\section{Appendix A:}

Proof of Proposition 1.

Proof. Partition applicants into three groups according to where they end up under the truthful outcome: those who get admitted to school 1 (with measure $s_{1}$ ), those who get admitted to school 2 (with measure $s_{2}$ ), and the rest. Call them group 1, group 2, and group 3 applicants, respectively. Given the policy pair $(i, j)$ in which at least one of $i$ and $j$ equals IA, consider the following strategies: each group 1 applicant specifies school 1 as their top choice, and each group 2 applicant specifies school 2 as top choice. (It does not matter what the rest will do.) We first note that school $k$ considers every group $k$ applicant strictly better than every group 3 applicant, $k=1,2$. (Otherwise, it should have given a place to some group 3 applicant under (DA,DA).) Therefore, in the first round of the Gale-Shapley mechanism, school $k$ will keep all group $k$ applicants. Each school that uses IA will simply admit this group of applicants and will not proceed to the second round. The school, if any, that uses DA will find the applicants it retained in the first round to be strictly better than any applicant whom the school can newly consider in the second round. As a result, the mechanism terminates with school $k$ admitting all group $k$ applicants, $k=1,2$. Given the aforementioned prescription for applicants, it is clear that no applicant can benefit from a unilateral deviation; otherwise, she would have benefitted from a unilateral deviation under (DA,DA). Thus, the truthful outcome is still feasible as an equilibrium outcome under $(i, j)$. To show that this is the unique equilibrium outcome, it suffices to note that the truthful outcome is stable and hence there do not exist an unmatched pair of school and an applicant that prefer one other more than what they are entitled to under the truthful outcome.

\section{Proof of Proposition 4}

Proof. Step 1: We first note that $n(A)=n(B)=n(C)$. Denote this quantity by $Q$. We also note that

$$
m(A)=\frac{1}{2}\left(\frac{s_{1}}{\mu_{1}^{1} N}+\frac{s_{1}}{\mu_{1}^{2} N}\right), m(B)=\frac{s_{1}+s_{2}}{N}-\frac{x}{2} \text { and } m(C)=\frac{s_{1}+s_{2}}{N}+\frac{x}{2} .
$$

Substituting all these into (8) and with some manipulation, we obtain

$$
\begin{aligned}
\Delta\left(\mu_{1}^{1}, \mu_{1}^{2}\right) & =\frac{\pi_{2} Q}{2}\left(\frac{s_{1}}{\mu_{1}^{1} N}+\frac{s_{1}}{\mu_{1}^{2} N}\right)-\pi_{2} Q\left(\frac{s_{1}+s_{2}}{N}\right)+Q \frac{s_{2}}{N}\left(1-\frac{\mu_{1}^{2}}{\mu_{1}^{1}}\right)\left(\pi_{1}+\frac{\pi_{2}}{2}\right) \\
& =\frac{s_{1} Q}{N}\left[\frac{\pi_{2}}{2}\left(\frac{1}{\mu_{1}^{1}}+\frac{1}{\mu_{1}^{2}}\right)-\pi_{2}\left(\frac{s_{1}+s_{2}}{s_{1}}\right)+\left(1-\frac{\mu_{1}^{2}}{\mu_{1}^{1}}\right)\left(\pi_{1}+\frac{\pi_{2}}{2}\right)\right],
\end{aligned}
$$

Step 2: $\partial \Delta\left(\mu_{1}^{1}, \mu_{1}^{2}\right) / \partial \mu_{1}^{2}<0$.

Step 3: Note that the lowest possible value of $\mu_{1}^{2}$ is just $s_{1} /\left(s_{1}+s_{2}\right)$. Substituting it 
into $\Delta\left(\mu_{1}^{1}, \mu_{1}^{2}\right)$, and after regrouping, yields

$$
\begin{aligned}
\Delta\left(\mu_{1}^{1}, \frac{s_{1}}{s_{1}+s_{2}}\right) & =\frac{s_{1} Q}{N}\left[\frac{\pi_{2}}{2}\left(\frac{1}{\mu_{1}^{1}}+\frac{s_{1}+s_{2}}{s_{1}}\right)-\pi_{2} \beta+\left(1-\frac{s_{1}}{s_{1}+s_{2}} \frac{1}{\mu_{1}^{1}}\right)\left(\pi_{1}+\frac{\pi_{2}}{2}\right)\right] \\
& =\frac{s_{1} Q}{N} \frac{\pi_{2}}{2}\left[\left(\frac{s_{2}}{s_{1}}-\frac{2 \pi_{1}}{\pi_{2}}\right)\left(\frac{1}{\mu_{1}^{1}}-\frac{s_{1}+s_{2}}{s_{1}}\right)\right] .
\end{aligned}
$$

Hence, $\Delta\left(\mu_{1}^{1}, s_{1} /\left(s_{1}+s_{2}\right)\right)>0$ if and only if (i) $s_{2} / s_{1}>2 \pi_{1} / \pi_{2}$ and $\mu_{1}^{1}<s_{1} /\left(s_{1}+s_{2}\right)$ (rejected) or (ii) $s_{2} / s_{1}<2 \pi_{1} / \pi_{2}$ and $\mu_{1}^{1}>s_{1} /\left(s_{1}+s_{2}\right)$.

Step 4: Using step 2 and step 3, as well as continuity, we establish that there exist parameters in which $\Delta\left(\mu_{1}^{1}, \mu_{1}^{2}\right)>0$ if $s_{2} / s_{1}<2 \pi_{1} / \pi_{2}$ and there do not if $s_{2} / s_{1} \geq 2 \pi_{1} / \pi_{2}$.

Step 5: In the above derivation, as well as the exposition in the main text, we assumed that the right boundary of region $\mathrm{A}$ is on the left hand side of the left boundary of region B. It may not be the case, and there may be "overlapping" of the two regions. It can be easily shown that, however, all the calculations in this proof, as well as the proposition, still go through. The trick is that we can add the overlapping region to both the gain and loss in state 2 so that the specifications of Region A and Region B that we used in defining $\Delta$ become without loss of generality whether or not there is overlapping of the two regions.

Proof of Proposition 5.

Proof. We now reckon how school 2's composition of admitted applicants is changed under (IA,IA) relative to (DA,IA). We first note that, during this exercise, in state 2, school 1 admits those type 1 applicants with $y_{1} \in V \equiv\left(\left(s_{1}+s_{2}\right) / N,\left(s_{1}+s_{2}\right) / N+x^{\prime}\right)$, replacing those type 2 applicants with $y_{1} \in T$. Here

$$
x^{\prime}=x\left(1 / \mu_{1}^{2}-1\right)
$$

Back to state 1, school 2 needs to replace those type 1 applicants with $y_{1} \in T$ by applicants with $y_{1}>\left(s_{1}+s_{2}\right) / N$. Those applicants will be solely of type 2 applicants if type 2 applicants with $y_{1} \in V$ are sufficiently numerous for the replacement and will be of both types otherwise.

Case 1: If the replacement is solely by type 2 applicants, then the replacement actually comes from type 2 applicants with $y_{1} \in\left(\left(s_{1}+s_{2}\right) / N,\left(s_{1}+s_{2}\right) / N+z\right)$ where

$$
z=x\left(1 / \mu_{2}^{1}-1\right)
$$

This case happens when $\mu_{1}^{2}<\mu_{2}^{1}$.

Case 2: If the replacement is by both types of applicants, then the replacement actually comes from type 2 applicants with $y_{1} \in\left(\left(s_{1}+s_{2}\right) / N,\left(s_{1}+s_{2}\right) / N+z\right)$ and type 1 applicants 
with $\left.y_{1} \in\left(\left(s_{1}+s_{2}\right) / N+x^{\prime},\left(s_{1}+s_{2}\right) / N+z\right)\right)$, where

$$
z-x^{\prime}=\mu_{1}^{1} x-\mu_{2}^{1} x^{\prime}
$$

This case happens when $\mu_{1}^{2}>\mu_{2}^{1}$.

Now return to $\Gamma\left(\mu_{1}^{1}, \mu_{1}^{2}\right)$. Noting that $n(A)=n(B)$, denoted by $Q$, and $n\left(B^{\prime}\right)=n(C)=$ $\mu_{1}^{1} n(B)$. Note also that

$$
m(A)=\frac{1}{2}\left(\frac{s_{1}}{\mu_{1}^{1} N}+\frac{s_{1}}{\mu_{1}^{2} N}\right), m\left(B^{\prime}\right)=m(b)=\frac{s_{1}+s_{2}}{N}-\frac{x}{2} \text { and } m(C)=\frac{s_{1}+s_{2}}{N}+R x,
$$

where $R$ is a positive term. We thus have

$$
\begin{aligned}
\Gamma\left(\mu_{1}^{1}, \mu_{1}^{2}\right) & =Q\left[\pi_{2} m(A)-\left(\pi_{2}+\pi_{1} \mu_{1}^{1}\right) m(B)+\pi_{1} \mu_{1}^{1} m(C)\right] \\
& =Q\left[\frac{\pi_{2}}{2}\left(\frac{s_{1}}{\mu_{1}^{1} N}+\frac{s_{1}}{\mu_{1}^{2} N}\right)-\left(\pi_{2}+\pi_{1} \mu_{1}^{1}\right)\left(\frac{s_{1}+s_{2}}{N}-\frac{x}{2}\right)+\pi_{1} \mu_{1}^{1}\left(\frac{s_{1}+s_{2}}{N}+R x\right)\right]
\end{aligned}
$$

Note that the lowest possible value of $\mu_{1}^{2}$ is just $s_{1} /\left(s_{1}+s_{2}\right)$. Substituting it into $\Delta\left(\mu_{1}^{1}, \mu_{1}^{2}\right)$, and after regrouping, yields

$$
\Gamma\left(\mu_{1}^{1}, \frac{s_{1}}{s_{1}+s_{2}}\right)=\left(-\frac{s_{1}+s_{2}}{s_{1}}+\frac{1}{\mu_{1}^{1}}\right)\left(\frac{s_{2}}{s_{1}}-\mu_{1}^{1}(1+2 R) \frac{\pi_{1}}{\pi_{2}}\right) .
$$

Hence, $\Delta\left(\mu_{1}^{1}, s_{1} /\left(s_{1}+s_{2}\right)\right)>0$ if and only if (i) $s_{2} / s_{1}>\mu_{1}^{1}(1+2 R) \pi_{1} / \pi_{2}$ and $\mu_{1}^{1}<$ $s_{1} /\left(s_{1}+s_{2}\right)$ (rejected) or (ii) $s_{2} / s_{1}<\mu_{1}^{1}(1+2 R) \pi_{1} / \pi_{2}$ and $\mu_{1}^{1}>s_{1} /\left(s_{1}+s_{2}\right)$. Since the second condition is always satisfied, we can just focus on the first one. Hence, $\Delta\left(\mu_{1}^{1}, s_{1} /\left(s_{1}+s_{2}\right)\right)>$ 0 if and only if

$$
\frac{s_{2}}{s_{1}}<\mu_{1}^{1}(1+2 R) \frac{\pi_{1}}{\pi_{2}} .
$$

If Case 1 is the case, then $R=z / 2 x$, (A1) becomes $s_{2} / s_{1}<\mu_{1}^{1} /\left(1-\mu_{1}^{1}\right) \pi_{1} / \pi_{2}$. If Case 2 is the case, then given $\mu_{1}^{1}$ and $\mu_{1}^{2}, R$ is bounded below by zero, and in particular $R>x^{\prime} / 2 x=\left(1 / \mu_{1}^{2}-1\right) / 2=\mu_{1}^{1} / 2\left(1-\mu_{1}^{1}\right)$. Hence, taken into account continuity, a sufficient condition for existence of the preemptive equilibrium is that $s_{2} / s_{1}<\mu_{1}^{1} /\left(1-\mu_{1}^{1}\right) \times \pi_{1} / \pi_{2}$.

\section{Proof of Proposition 7.}

Proof. To see this, suppose that all other applicants report truthfully. Then each applicant is admitted by school 1 with $s_{1} / N$ probability and by school 2 with $s_{2} / N$ probability, obtaining an expected utility of $s_{1} u_{1} / N+s_{2} u_{2} / N$. By unilaterally deviating to choosing school 2 as her top choice, the applicant is certain to be admitted by school 2, obtaining a utility of $u_{2}$. If $u_{2} / u_{1}<s_{1} /\left(N-s_{2}\right)$, such a deviation is unprofitable and the truthful equilibrium remains to be viable-school 2's IA is simply irrelevant. If $u_{2} / u_{1}>s_{1} /\left(N-s_{2}\right)$, 
on the other hand, the deviation is profitable and the truthful equilibrium is not viable. Instead, the equilibrium is characterized by a pair, $a$ and $b \equiv N-a$, where $a(b)$ is the number of applicants making school 1 (2) their top choice. Intuitively, the higher $u_{2} / u_{1}$, the greater the equilibrium $b$ is. Moreover, when $b$ is sufficiently large, the use of IA by school 2 indeed improves the average quality of its applicants.

Here we prove 3 ; the other parts are straightforward. We first note that, in equilibrium, school 1's cutoff value must be more stringent than school 2's cutoff value; or else, each applicant would strictly prefer to make school 1 her top choice, violating the fact that school 1's cutoff value is less stringent. This implies that the applicant who makes school 2 her top choice and is subsequently declined by school 2 will not be admitted by school 1 . We next note that $b$, the number of applicants making school 2 their top choice, must exceed $s_{2}$. (Suppose not. Then, by making school 2 the top choice, an applicant is certain to receive a utility of $u_{2}$-she is admitted by school 2 for certain. By making school 1 her top choice, the applicant is admitted by school 1 with probability $p=s_{1} /(N-b)$ and by school 2 with probability $q=(1-p)\left(s_{2}-b\right) /\left(N-s_{1}-b\right)$. The equilibrium dictates that $u_{2}=p u_{1}+q u_{2}$, or $u_{2} / u_{1}=s_{1} /\left(N-s_{2}\right)$, which is contradictory.) Given these two facts, we learn that, in equilibrium, no applicant will be admitted by a school that she did not specify as her top choice. Hence, indifference between the two strategies means: $s_{2} u_{2} / b=s_{1} u_{1} /(N-b)$, implying

$$
b=\frac{s_{2} u_{2} N}{s_{1} u_{1}+s_{2} u_{2}} .
$$

To show the last result, we first note that $A Q_{2}(D A, I A)=\left(s_{1} u_{1}+s_{2} u_{2}\right) / 2 u_{2} N$. Under (DA,DA), all applicants choose school 1 as their top choice. Then, school 1 admits those applicants with $y_{1}<c_{1} \equiv s_{1} / N$ and school 2 admits those applicants with $<y_{1} \in\left(c_{1}, c_{2}\right)$ where $c_{2} \equiv\left(s_{1}+s_{2}\right) / N$. As a result, $A Q_{2}(D A, D A)=\left(2 s_{1}+s_{2}\right) / 2 N$, which is strictly greater than $A Q_{2}(D A, I A)$ if and only if $u_{2} / u_{1}>1 / 2$.

\section{Proof of Proposition 9.}

Proof. We first note that it must be the case that $b>s_{2}$. Suppose not. By specifying school 1 as top choice, an applicant is admitted to school 1 with probability $s_{1} / a$ and to school 2 with probability $\left(s_{2}-b\right) / a$, resulting in a utility of $s_{1} u_{1} / a+\left(s_{2}-b\right) u_{2} / a$. By specifying school 2 as her top choice, an applicant is certain to be admitted to school 2 and obtain a utility of $u_{2}$. But

$$
u_{2}>\frac{s_{1}}{a} u_{1}+\frac{s_{2}-b}{a} u_{2} \Leftrightarrow \frac{u_{2}}{u_{1}}>\frac{s_{1}}{N-s_{2}} .
$$

Thus everybody indeed wants to make school 2 the top choice, and this is contradictory to the claim that, in equilibrium, $b \leq s_{2}$. Given that $b>s_{2}$, it is easy to reckon that school 1 will set a cutoff value of $c_{1}=s_{1} /\left(N-s_{2}\right)$. By reporting 1 as her top choice, an applicant is 
admitted to school 1 with probability $c_{1}$ and to school 2 with probability zero; by reporting 2 as her top choice, the applicant is admitted to school 2 with probability $s_{2} / b$ and to school 1 with probability $\left(1-s_{2} / b\right) c_{1}$. Hence, making 2 as the top choice is strictly better if and only if

$$
c_{1} u_{1}<\frac{s_{2}}{b} u_{2}+\left(1-\frac{s_{2}}{b}\right) c_{1} u_{1} \Leftrightarrow \frac{u_{2}}{u_{1}}>c_{1}=\frac{s_{1}}{N-s_{2}},
$$

suggesting that, in equilibrium, $b=N$ !

Proof of Proposition 10.

Proof. Under (IA,IA) in equilibrium, the numbers of applicants who specify school 1 and who specify school 2 as their top choice are $a=s_{1} u_{1} /\left(s_{1} u_{1}+s_{2} u_{2}\right)>s_{1}$ and $b=$ $s_{2} u_{2} /\left(s_{1} u_{1}+s_{2} u_{2}\right)>s_{2}$, respectively. Moreover, the average qualities of the admitted applicants are

$$
A Q_{1}(I A, I A)=\frac{s_{1} u_{1}+s_{2} u_{2}}{2 u_{1}} \text { and } A Q_{2}(I A, I A)=\frac{s_{1} u_{1}+s_{2} u_{2}}{2 u_{2}} .
$$

By simple comparison, we reckon that $A Q_{1}(D A, I A)<A Q_{1}(I A, I A)$ and $A Q_{2}(I A, D A)<$ $A Q_{2}(I A, I A)$. 